\title{
Spectral resolution and coverage impact on advanced sounder information content
}

\author{
Allen M. Larar ${ }^{1}$, Xu Liu ${ }^{1}$, Daniel K. Zhou ${ }^{1}$, William L. Smith, ${ }^{2,3}$ \\ ${ }^{1}$ NASA Langley Research Center, Hampton, VA, USA \\ ${ }^{2}$ Hampton University, Hampton, VA, USA \\ ${ }^{3}$ University of Wisconsin-Madison, Madison, WI, USA
}

\begin{abstract}
Advanced satellite sensors are tasked with improving global measurements of the Earth's atmosphere, clouds, and surface to enable enhancements in weather prediction, climate monitoring capability, and environmental change detection. Achieving such measurement improvements requires instrument system advancements. This presentation focuses on the impact of spectral resolution and coverage changes on remote sensing system information content, with a specific emphasis on thermodynamic state and trace species variables obtainable from advanced atmospheric sounders such as the Infrared Atmospheric Sounding Interferometer (IASI) and Cross-track Infrared Sounder (CrIS) systems on the MetOp and NPP/NPOESS series of satellites.
\end{abstract}

Key words: remote sensing, advanced sounders, information content, IASI, CrIS 